\title{
La toxicología en la vida económica, política y social de nuestros países*
}

\author{
Toxicology in the economic, political and social life of our \\ countries
}

\section{Rafael Pérez Cristiá}

Miembro Titular de la Academia de Ciencias de Cuba. Buró Regulatorio para la protección de la salud. La Habana, Cuba.

Distinguidos delegados e invitados

Estimados colegas

Es un alto honor para mí la invitación que me hiciera la Sociedad Cubana de Toxicología y el Comité Organizador para presentar la Conferencia Magistral de este IV Congreso Internacional de Toxicología, además de significar su aceptación para mí un verdadero reto por su contenido al pretender enfocar desde mi perspectiva aspectos de la relevancia económica, política y social que tiene la toxicología contemporánea en nuestros países.

Las sustancias químicas son indispensables en la vida del hombre, contribuyen al aumento de la producción de alimentos, alivian muchas enfermedades y facilitan la existencia cotidiana de nuestra sociedad. Todos los años aparecen nuevos productos con ventajas superiores sobre los existentes y con una gran viabilidad comercial.

La preocupación por la protección de la salud humana contra los efectos perjudiciales que provocan una gran cantidad y variedad de productos se ha puesto 
de manifiesto prácticamente en todos los países. El comercio internacional de muchos productos abarca a todo el mundo e involucra cada vez más a países en desarrollo, donde la legislación, cuando existe, suele ser incompleta o se aplica de forma esquemática y sin rigor.

El desarrollo de la toxicología como una ciencia independiente se ha ido consolidando durante los últimos años ante la necesidad de profundizar los conocimientos para abordar los problemas toxicológicos y de la contaminación ambiental que afectan nuestro planeta y la extraordinaria revolución que ha provocado en la ciencia el desarrollo de la biología molecular y el conocimiento de los procesos biológicos.

A lo anterior debemos agregar el imponente desarrollo que ha experimentado la industria química, imprescindible en una sociedad moderna caracterizada por su alta tecnología, la cual ha determinado la necesidad de que muchos países presten mayor atención al desarrollo de la toxicología.

Según reportes, la industria química de América Latina representa alrededor del 4 $\%$ del mercado mundial y no ha crecido al ritmo que lo hacen otros países en especial los de economías emergentes, siendo Brasil, México y Argentina, en ese orden, los principales productores de químicos en lo general. Sin embargo, e independientemente de lo anterior, cada día aumenta más el volumen de sustancias químicas que generan impacto ambiental y crean condiciones de mayor exposición a contaminantes y residuos peligrosos en nuestra región.

Ejemplo de ello son el uso intensivo e indiscriminado de los productos fitosanitarios en nuestros países, la mayoría de ellos en desuso o prohibidos en los países desarrollados, las emisiones de sustancias químicas contaminantes a la atmósfera que genera la industria del petróleo y sus derivados, la contaminación por la actividad minera, la exposición a plomo, mercurio y arsénico, el fenómeno invernadero, la deforestación y una lista interminable de otros problemas.

Los episodios tóxicos de toda índole constituyen en nuestra sociedad de hoy una preocupación global, dado por la utilización abusiva y no reglamentada en nuestros países de las sustancias químico-tóxicas, de los productos confeccionados con las mismas y de los desechos peligrosos que resultan de su empleo, lo cual impacta brutalmente y genera colosales problemas que afectan la salud del hombre y el medio ambiente y atentan muy seriamente contra la naturaleza y la existencia propia de la especie humana.

El modelo neoliberal actual que pretende imponerse de forma global, cuya economía es regulada por las fuerzas del mercado, hace que las probabilidades de proteger efectivamente el medio ambiente sean realmente bajas, y determina que la sociedad actué en forma dispersa y poco coordinada para abordar los verdaderos determinantes del deterioro o preservación ambiental, que son fundamentalmente de tipo político, económico y tecnológico. La salud y el ambiente no pueden ser regulados exclusivamente por las fuerzas del mercado.

Lamentablemente en estas condiciones no se ha podido generar la información necesaria para demostrar que los problemas de salud asociados a fenómenos tóxicos y ambientales son también de importancia relevante como lo son otros problemas emergentes de salud, de hecho, hoy existe una mayor cantidad de personas expuestas a niveles de riesgo por la contaminación atmosférica, del agua y del suelo y sus consecuentes problemas de salud en América Latina. 
El uso inadecuado de sustancias químicas se ha incrementado en la región así como su relación con el daño a la salud de la población, el daño ecológico y las consecuencias sociales y económicas sobre el desarrollo, lo cual ha creado una grave situación que compromete tanto el desarrollo de los países en general como a la Salud Pública en especial así como la potencial ocurrencia de accidentes químicos.

Los riesgos que se derivan de la exposición y la incidencia creciente a nivel mundial de enfermedades asociadas a las sustancias tóxicas reclama de los gobiernos de cada país una mayor atención y la adopción de políticas claras y definidas que permitan armonizar el rol de los diversos sectores en la prevención y el manejo integral de estos problemas.

Resulta muy conveniente también, que unido a los diferentes tipos de investigaciones toxicológicas, se realicen estudios epidemiológicos encaminados a conformar y avalar los datos experimentales y al mismo tiempo estudiar la acción de las sustancias tóxicas en el medio ambiente y las consecuencias que provocan sobre el hombre, las plantas y los animales.

Todo ello hace impostergable la adopción en cada país de programas específicos multisectoriales para prevenir y controlar, mediante el fortalecimiento de la legislación y la reglamentación, los riesgos que representan las sustancias químicas para la salud y el ambiente.

Muchos países en el mundo y en nuestra región han desarrollado modificaciones administrativas y estructurales a nivel nacional para elevar la categoría de las dependencias de regulación, control y vigilancia encargadas de la protección de la salud ambiental y han incrementado los recursos humanos que se desempeñan en esta área.

Se está produciendo un cambio de paradigma que va del esquema esencialmente administrativo de la regulación sanitaria a la visión científica de la protección de la población contra riesgos sanitarios. Estos cambios estratégicos están orientados a fortalecer el papel rector de la salud con un enfoque administrativo y de desempeño orientado a la regulación, control y fiscalización en función de la salud pública y su impacto sobre la morbilidad y mortalidad de nuestra población.

Esta nueva estrategia de regulación sanitaria incluye a la toxicología regulatoria y el fortalecimiento de la Red de Centros de Información y Asesoramiento Toxicológico, Control de Intoxicaciones y Centros de Toxicología, como parte de las autoridades reguladoras sanitarias competentes en los países y como un componente fundamental en regulación, control y fiscalización de las sustancias tóxicas o peligrosas, tales como plaguicidas, fertilizantes, precursores químicos, químicos esenciales y otros relacionados y en la toxicovigilancia como proceso activo de identificación y evaluación de los riesgos tóxicos en la comunidad, con el objetivo de reducirlos o eliminarlos así como la evaluación de las medidas tomadas para reducir los riesgos identificados.

Estas medidas han dado lugar al surgimiento de las Agencias Nacionales de Vigilancia Sanitaria y Protección de la Salud en nuestra región entre las que se destacan el Instituto Nacional de Vigilancia de Medicamentos y Alimentos (INVIMA) de Colombia, la Administración Nacional de Medicamentos, Alimentos y Tecnologías Médicas (ANMAT) de Argentina, y el Buró Regulatorio para la Protección de la Salud de Cuba; la Agencia Nacional de Vigilancia Sanitaria (ANVISA) de Brasil y la Comisión Federal de Protección contra Riesgos Sanitarios (COFEPRIS) de México, 
estas últimas han incorporado a la toxicología como parte de sus estructuras y funciones.

El fortalecimiento de la capacidad nacional para garantizar el control y la vigilancia sanitaria de todos los productos que puedan tener una influencia nociva sobre la salud humana como parte integrante del programa para el perfeccionamiento de la organización, planificación y administración estratégica de los servicios de salud y el perfeccionamiento de la política de regulación sanitaria nacional, constituyen objetivos de trabajo, actuales y perspectivos, del Ministerio de Salud Pública de Cuba.

Con estas premisas se crea por el citado ministerio en el año 1996 el Buró Regulatorio para la Protección de la Salud (BRPS) cuya misión principal es mantener la vigilancia sanitaria y proteger la salud de la población garantizando la calidad, seguridad, eficacia y efectividad de los productos y servicios de salud.

De cualquier manera y en el más amplio de los sentidos, la toxicología cubana ha jugado un papel fundamental en el impulso al desarrollo de las principales actividades científicas, productivas, legales y de salud en nuestro país, desde la toxicología experimental a la toxicología forense, desde una perspectiva científica a otra regulatoria, desde la toxicología analítica a la social, en resumen, la toxicología como disciplina ha acompañado nuestro desarrollo social.

Habiendo expresado lo anterior y tomando en cuenta que de cualquier manera el tema social se halla en el centro del escenario histórico de América Latina y el incremento de la magnitud y profundidad de los problemas sociales que impactan en la región, los invito a reflexionar en lo que pudiéramos hipotéticamente denominar como una nueva rama de la toxicología, la toxicología encargada del estudio de los problemas que envenenan y matan a nuestros pueblos.

Los niveles de pobreza absoluta siguen aumentando, nunca antes había un número similar de pobres. Se estima que son 1,3 billones de seres humanos que sobreviven con menos de un dólar al día, desempleo e informalidad del empleo, incluida explotación infantil, carencias de todo tipo en materia de salud pública, falta de educación, erosión de la familia, aumento de la delincuencia, la criminalidad, la exclusión y los niveles de desigualdad absoluta. Reflexionemos y actuemos.

Quiero concluir esta conferencia con un agradecimiento a los organizadores de este congreso por haberme dado la oportunidad de compartir con ustedes algunos puntos de vistas de la toxicología, su desarrollo, principales problemas y perspectivas futuras, temas que sin duda, estarán presentes en los debates del congreso.

Muchas gracias.

Recibido: 6 de diciembre de 2007.

Aprobado: 7 de diciembre de 2007. 
Rafael Pérez Cristiá. Buró Regulatorio para la protección de la salud. La Habana, Cuba. E-mail: cecmed@infomed.sld.cu

*Conferencia presentada en el IV Congreso Internacional de Toxicología. La Habana. 4-7 de diciembre de 2007. 